\title{
Mycoplasma Pneumoniae Associated Transverse Myelitis in a 26-Year-Old Male
}

\author{
Marina Kontogiorgi ${ }^{\mathrm{a}}$, Grigoris Stratakos ${ }^{\mathrm{b}}$, Anastasia Kotanidou $^{\mathrm{a}}$, Antonios Tavernarakis ${ }^{\mathrm{c}}$, \\ Demetrios Exarhos ${ }^{\mathrm{d}}$, Christina Routsi ${ }^{\mathrm{a}, \mathrm{e}}$
}

\begin{abstract}
Transverse myelitis is a rare, serious complication of Mycoplasma pneumoniae infection. Here we report a case of acute transverse myelitis in a previously healthy 26 -year-old male, during the course of a lower respiratory infection. The diagnosis was based on magnetic resonance imaging findings of the spinal cord and on the quantitative determination of $\operatorname{IgM}$ and $\operatorname{IgG}$ antibodies against M. pneumoniae in the serum and a positive nested PCR test in the sputum. Diagnostic workout did not reveal any other cause of the neurological symptoms. The patient was treated successfully with intravenous azithomycin and doxycycline. His recovery was progressive and he was discharged without any neurologic sequelae.
\end{abstract}

Keywords: Mycoplasma pneumoniae; Acute transverse myelitis; Respiratory infection; Neurological complication

\section{Introduction}

Mycoplasma pneumoniae is a common cause of community associated upper and lower respiratory tract infections most commonly in children and young adults [1]. Previously thought to cause only benign respiratory disease, it can also cause severe disease and is occasionally associated with extrapulmonary complications [1-3]. Among them, neurological complications of $M$. pneumoniae infection were first

\footnotetext{
Manuscript accepted for publication August 13, 2013

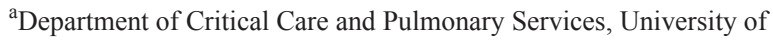
Athens Medical School, Evangelismos Hospital, Athens, Greece

${ }^{\mathrm{b}}$ Department of Respiratory Medicine, University of Athens Medical School, Sotiria Hospital, Athens, Greece

${ }^{\mathrm{c}}$ Department of Neurology, Evangelismos Hospital, Athens, Greece

${ }^{\mathrm{d}}$ Department of Radiology, Evangelismos Hospital, Athens, Greece

${ }^{\mathrm{e} C o r r e s p o n d i n g ~ a u t h o r: ~ R o u t s i ~ C h r i s t i n a, ~ D e p a r t m e n t ~ o f ~ C r i t i c a l ~ C a r e, ~}$

Evangelismos Hospital, $45-47$ Ipsilantou Str, Athens 106 76, Greece.

Email: chroutsi@hotmail.com
}

doi: http://dx.doi.org/10.4021/jmc1480e been described in the 60's [4] and is believed to have an estimated incidence of $0.1 \%$ in all patients with a $M$. pneumoni$a e$ infection and up to $7 \%$ of those with more severe disease requiring hospitalization [1-3]. Encephalitis with or without meningeal involvement is the most frequent, followed by aseptic meningitis, polyradiculitis, Guillain-Barre syndrome, stroke, cerebral infarction, cerebral ataxia, and acute transverse myelitis [1-3]. The first case of acute transverse myelitis in association with $M$. pneumoniae infection was reported in 1973 as a postmortem diagnosis [5]. Up to the year 2006, only 35 cases of $M$. pneumoniae-associated acute transverse myelitis had been reported worldwide, followed by some additional single cases. Among them, only 18 were serologically proven [6]. We present another case of acute transverse myelitis that occurred in a young male, during the course of a respiratory tract infection from M. pneumoniae. The purpose of this report is to serve as a reminder of this rare complication of $M$. pneumoniae infection.

\section{Case Report}

A 26-year-old smoking (8 pack-years) male presented to the emergency department with a 15-day history of respiratory tract illness with a sore throat, productive cough, headache, and fever up to $39^{\circ} \mathrm{C}$. He had been initially diagnosed as an upper respiratory tract bacterial infection, based on pharyngeal hyperemia and tonsillar exudates that were detected by a private physician, and he was treated with amoxicillin/clavulanate without significant improvement. His medical and family histories were unremarkable. He was a construction worker and he had not traveled recently.

On physical examination he appeared to be in mild respiratory distress. His heart rate was 120 beats per minute, respiratory rate was 40 breaths per minute, body temperature $38^{\circ} \mathrm{C}$, blood pressure $90 / 50 \mathrm{~mm} \mathrm{Hg}$, and his pulse oxymetry oxygen showed a saturation rate of $94 \%$ in room air. Arterial blood gas measurement revealed a $\mathrm{pH}$ of 7.54 , a $\mathrm{PCO}_{2}$ of 25 $\mathrm{mm} \mathrm{Hg}$, and a $\mathrm{PO}_{2}$ of $60 \mathrm{~mm} \mathrm{Hg}$.

Chest auscultation revealed scattered rhonchi, prolonged expiration and rales localized to the left lung base. The remainder of the physical examination was unremarkable. 


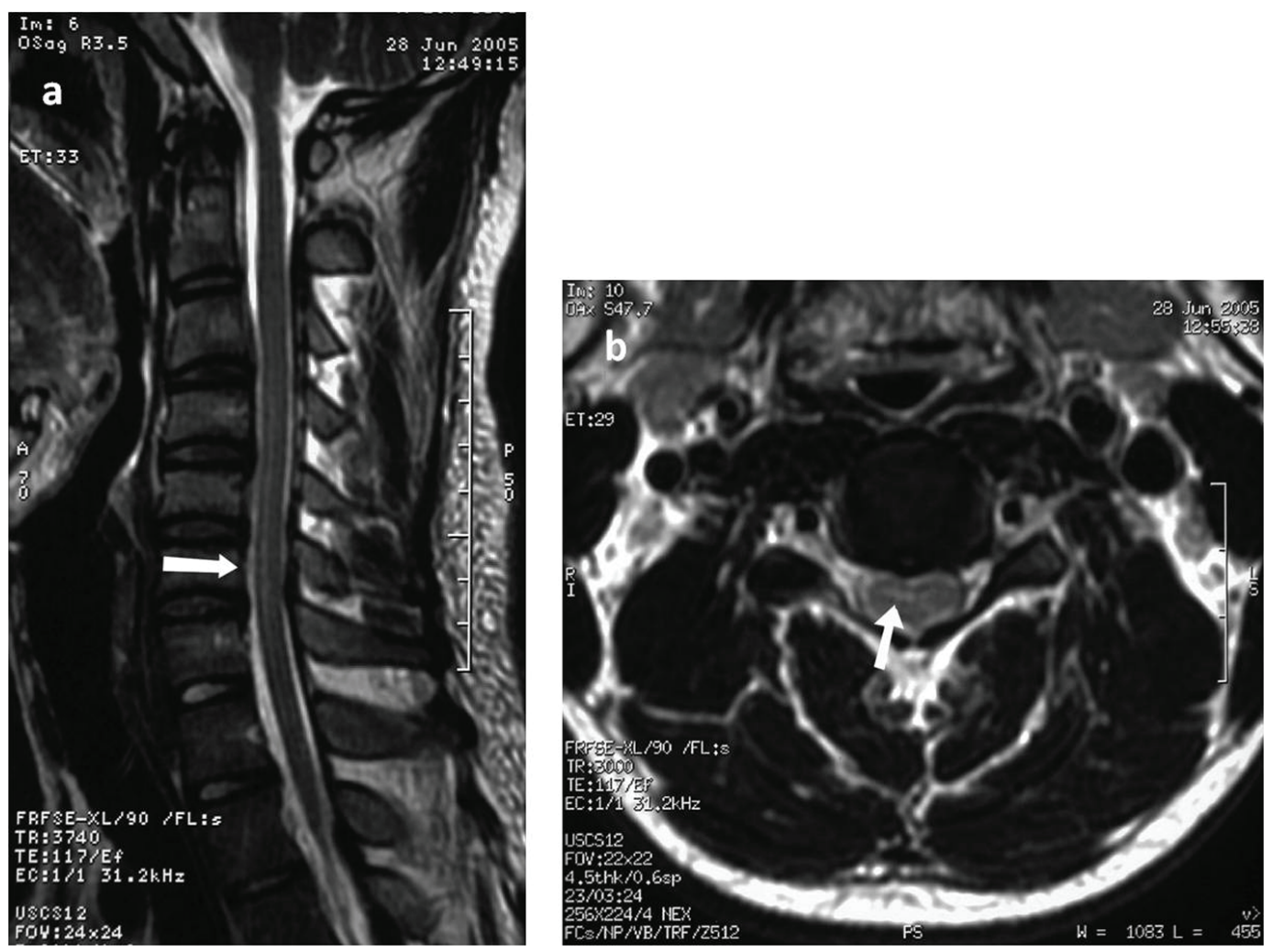

Figure 1. Sagittal T2-weighted magnetic resonance imaging of the cervical spine exhibiting increased medullary signal intensity over C3 to C6 (a) and T2-weighted axial scan at the level of the cervical spine showing the intramedullary high signal intensity(b).

Initial laboratory findings showed leucocytosis (total white cell count $18.9 \times 10^{9} / \mathrm{L}$ ) and an erythrocyte sedimentation rate of $93 \mathrm{~mm} /$ hour; blood chemistry analysis was within normal limits with the exception of a C-reactive protein of $20 \mathrm{mg} / \mathrm{dL}$. A chest X-ray revealed an infiltrate in the left lung. Chest computed tomography revealed a pulmonary consolidation with an air-bronchogram at the lower aspect of the lingula. The diagnosis of pneumonia was made and the patient was admitted to the pulmonary department. After collection of sputum and blood samples for bacteriologic and serological testing, antimicrobial treatment was started including clarithromycin $500 \mathrm{mg} \times 2$ per os and amoxycillin $500 \mathrm{mg} \times 4 \mathrm{IV}$.

Two days after his hospital admission the patient developed ascending paresthesias in his lower extremities, progressive leg weakness, gait difficulty and urinary retention requiring bladder catheterization. Deep tendon reflexes of the lower extremities were symmetrically brisk and sensation was intact. At that time he was afebrile and normotensive. A lumbar puncture was performed; cerebrospinal fluid (CSF) analysis revealed a WBC count of $69 / \mathrm{mm}^{3}$ (lymphocytes $94 \%$ ), glucose of $67 \mathrm{mg} / \mathrm{dL}$, and a protein value of $79.5 \mathrm{mg} /$ $\mathrm{dL}$. Magnetic resonance imaging (MRI) of the spinal cord revealed an intramedullary lesion in the spinal cord with a high T2-W signal in the thoracic spinal cord extending at least three vertebral segments (C3 to C6). No cord swelling was detected (Fig. 1). Electromyography was unremarkable.

A thorough search for bacterial infection was negative. Sputum specimen culture as well as cultures of blood and CSF was negative. Serum testing for HIV and hepatitis B and $\mathrm{C}$ were negative. Antibodies for Cytomegalovirus, EpsteinBarr virus, Varicella zoster virus, Herpes simplex virus, Enteroviruses, Coxsackie B1-B6, Coxiella burnetti, Legionella pneumophila, Borrelia burgdorferi, Brucella and Chlamydia antibodies were negative. However, IgG, IgM and IgA antibodies for M. pneumoniae were detected at a concentration of $951 \mathrm{U} / \mathrm{mL}, 67 \mathrm{U} / \mathrm{mL}$ and $49 \mathrm{U} / \mathrm{mL}$ respectively. Detection of $M$. pneumoniae DNA in CSF and blood serum samples using a nested polymerase chain reaction method was negative whereas it was positive in the sputum. Mycoplasma-related acute transverse myelitis was provisionally diagnosed and the patient was moved to an intermediate intensive care unit for better monitoring and supportive care. Azithromycin 500 $\mathrm{mg} \times 2$ given intravenously and doxycycline $100 \mathrm{mg} \times 2$ was started. The patient's neurological status improved progressively and was discharged after a total hospital stay of 18 days. Three years after the initial episode the patient remains asymptomatic. 


\section{Discussion}

Acute transverse myelitis is characterized by focal inflammation of the spinal cord with resultant neural involvement. It is a rare disorder with an estimated incidence of $1-8$ cases per million of population per year [7] and may be caused by a variety of mechanisms. M. pneumoniae infection as a cause of acute transverse myelitis is a rare clinical entity, known from a limited number of discrepant cases until now, mainly reported in pediatric patients, since children are most likely affected [3-6].

Typically, the onset of $M$. pneumoniae-associated myelitis occurs during or after the course of the respiratory infection, usually within three weeks after the onset of respiratory illness. Largely consistent with this progress, the onset of acute transverse myelitis in our patient occurred on the seventeenth day after initiation of the respiratory illness symptoms, while he was recovering from pneumonia. However, there are reports of $M$. pneumoniae-associated acute transverse myelitis with no evidence of respiratory disease indicating the difficulty to suspect the infective cause of this neurological condition in such cases [8].

The diagnosis of acute transverse myelitis in our patient was based on the clinical picture, CSF findings and spinal neuroimaging results [9]. The laboratory diagnosis of $M$. pneumoniae infection was made by determination of specific antibodies in serum samples combined with detection of $M$. pneumoniae in a sputum specimen by nested PCR, as recommended [10]. Therefore, the patient fulfils criteria for a "probable" case of $M$. pneumoniae-related acute transverse myelitis according to the classification proposed by Tsiodras et al in a recent literature review [6]. Notably, only 2 out of the 35 reported cases with $M$. pneumoniae-associated acute transverse myelitis applied to this category whereas the remaining cases were classified as either "possible" or "suspected" or "indeterminate" [6].

Detection of M. pneumoniae by PCR or culture in CSF failed in the present case which is in accordance with other reports. Isolation of M. pneumoniae by culture in a CSF sample has never been reported in M. pneumoniae-associated myelitis in contrast to encephalitis cases [11]. Also, in only 2 cases PCR testing for M. pneumoniae in CSF was positive $[8,12]$. Early antimicrobial treatment, low sensitivity of the culture and poor specific serological response of the patients may be responsible for this.

The evidence of a causal association of transverse myelitis and $M$. pneumoniae respiratory infection is based mainly on the reported cases and on retrospective studies $[6,13]$. The pathogenetic mechanisms are not completely understood. Although direct invasion and the related host immune response are thought to be responsible for the pulmonary disease, the pathophysiological basis for the CNS manifestations is not elucidated. The theory of a direct invasion is not fully supported since isolation of $M$. pneumoniae by culture in a CSF sample has never been reported in M. pneumoniaeassociated myelitis; however, it cannot be ruled out since positive PCR testing for $M$. pneumoniae has been detected in CSF specimens on some occasions as mentioned above [8, 12]. It had been postulated that an immune-mediated process or an autoimmune reaction possibly play part in pathogenesis. Neuropathological observation of demyelination and perineural inflammation in cases with acute disseminated encephalomyelitis, give support to the latter suggestion. Importantly, among 49 children with M. pneumoniae infection, in two patients with acute cerebellar dysfunction, antibodies to mitotic spindle apparatus have been found indicating their role in the pathogenesis [14].

Macrolide or tetracycline classes are considered effective for treating M. pneumoniae infections. However, data on their concentrations in the cerebrospinal fluid are limited. Clarithromycin levels have been shown above the in vitro minimum inhibitory concentrations (MICs) for M. pneumoniae in CSF [15]; doxycycline also seems to reach therapeutic concentrations in CSF [16]. Notably, our patient was already under macrolide antimicrobial therapy at the onset of neurological symptoms. The administration of intravenous azithromycin and the addition of doxycycline might have contributed to his treatment since it allows sufficient concentrations in cerebrospinal fluid. However, the role of antibiotics remains questionable. Due to the possible immunological etiology of M. pneumoniae associated acute transverse myelitis, antibiotic treatment probably has no role. Therefore, in addition to antimicrobial therapy, immunomodulating therapies such as corticosteroids and intravenous immunoglobulin have been used with good results. However, there is insufficient evidence to show conclusively that they are effective. Some patients also had recovered without receiving such a treatment. Similarly, our patient had a successful recovery although he received only antimicrobial treatment.

Outcome was generally favorable in the reported cases of M. pneumoniae-associated acute transverse myelitis with the exception of two cases appearing in childhood and adolescence respectively and leading to persistent paraplegia $[17,18]$.

In summary, the current case demonstrates the sudden development of the rare entity of acute transverse myelitis in a patient with lower respiratory tract infection and extents the list of similar cases documented by both positive M. pneumoniae serology and by PCR from sputum. Given the high prevalence of $M$. pneumoniae respiratory infections, physicians evaluating patients with acute transverse myelitis neurologic symptoms, should have a high index of suspicion for $M$. pneumoniae-associated nervous system disease in order to optimize the management of such conditions.

\section{References}

1. Cassell GH, Cole BC. Mycoplasmas as agents of human 
disease. N Engl J Med. 1981;304(2):80-89.

2. Guleria R, Nisar N, Chawla TC, Biswas NR. Mycoplasma pneumoniae and central nervous system complications: a review. J Lab Clin Med. 2005;146(2):55-63.

3. Tsiodras S, Kelesidis I, Kelesidis T, Stamboulis E, Giamarellou H. Central nervous system manifestations of Mycoplasma pneumoniae infections. J Infect. 2005;51(5):343-354.

4. Endtz LJ, Hers JF. Mycoplasma pneumoniae polyradiculitis. Lancet. 1970;1(7642):358.

5. Nagaswami S, Kepes J, Foster DB, Twemlow SW. Necrotizing myelitis: a clinico-pathologic report of two cases associated with diplococcus pneumoniae and mycoplasma pneumoniae infections. Trans Am Neurol Assoc. 1973;98:290-292.

6. Tsiodras S, Kelesidis T, Kelesidis I, Voumbourakis K, Giamarellou H. Mycoplasma pneumoniae-associated myelitis: a comprehensive review. Eur J Neurol. 2006;13(2):112-124.

7. Pidcock FS, Krishnan C, Crawford TO, Salorio CF, Trovato M, Kerr DA. Acute transverse myelitis in childhood: center-based analysis of 47 cases. Neurology. 2007;68(18):1474-1480.

8. Abele-Horn M, Franck W, Busch U, Nitschko H, Roos R, Heesemann J. Transverse myelitis associated with Mycoplasma pneumoniae infection. Clin Infect Dis. 1998;26(4):909-912.

9. Proposed diagnostic criteria and nosology of acute transverse myelitis. Neurology. 2002;59(4):499-505.

10. Daxboeck F, Krause R, Wenisch C. Laboratory diagnosis of Mycoplasma pneumoniae infection. Clin Microbiol Infect. 2003;9(4):263-273.

11. Abramovitz P, Schvartzman P, Harel D, Lis I, Naot Y.
Direct invasion of the central nervous system by Mycoplasma pneumoniae: a report of two cases. J Infect Dis. 1987;155(3):482-487.

12. Goebels N, Helmchen C, Abele-Horn M, Gasser T, Pfister HW. Extensive myelitis associated with Mycoplasma pneumoniae infection: magnetic resonance imaging and clinical long-term follow-up. J Neurol. 2001;248(3):204-208.

13. Fernald GW. Immunologic mechanisms suggested in the association of M. pneumoniae infection and extrapulmonary disease: a review. Yale J Biol Med. 1983;56(56):475-479.

14. Cimolai N, Mah D, Roland E. Anticentriolar autoantibodies in children with central nervous system manifestations of Mycoplasma pneumoniae infection. J Neurol Neurosurg Psychiatry. 1994;57(5):638-639.

15. Maniu CV, Hellinger WC, Chu SY, Palmer R, AlvarezElcoro S. Failure of treatment for chronic Mycobacterium abscessus meningitis despite adequate clarithromycin levels in cerebrospinal fluid. Clin Infect Dis. 2001;33(5):745-748.

16. Karlsson M, Hammers S, Nilsson-Ehle I, Malmborg AS, Wretlind B. Concentrations of doxycycline and penicillin $G$ in sera and cerebrospinal fluid of patients treated for neuroborreliosis. Antimicrob Agents Chemother. 1996;40(5):1104-1107.

17. Pfausler B, Engelhardt K, Kampfl A, Spiss H, Taferner E, Schmutzhard E. Post-infectious central and peripheral nervous system diseases complicating Mycoplasma pneumoniae infection. Report of three cases and review of the literature. Eur J Neurol. 2002;9(1):93-96.

18. Csabi G, Komaromy H, Hollody K. Transverse myelitis as a rare, serious complication of Mycoplasma pneumoniae infection. Pediatr Neurol. 2009;41(4):312-313. 